Calderón, una ilusión. Ambos actos -el soñar de Campuzano y el despertar de Peralta- empiezan y terminan simultáneamente.

La perspectiva de la simultaneidad formal y metafísica continúa. Se dirigen Ios amigos juntos al Espolón a recrear los ojos del cuerpo, puesto que ya recrearon los del alma. Al respecto dice Avalle-Arce": "Cuando Campuzano y Peralta se marchan a pasear al Espolón de Valladolid, y así se salen del marco de las dos novelas (¿para entrar en la vida?), marchan unidos por la experiencia, pero separados por la verdad": Esta interpretación, así expuesta, es inexacta. Salen del marco novelístico unidos, absolutamente unidos, por la verdad, por la misma verdad que al principio - como ya he dicho- fue rechazada, burlada pero aceptada por fin sin reservas por el incrédulo licenciado. Dicha unión conseguida al final cierra el proceso vital de engaño y desengaño desarrollado en las dos obras que quedan por lo mismo fundidas en una sola. Por otra parte, esa unión en la verdad cierra perfectamente la simetría moral iniciada al principio de El casamiento, cuando Campuzano desde lejos ve a su amigo venir hacia él. Afirmando lo contrario a lo dicho por Avalle-Arce se tiene entonces que han entrado en el marco novelístico separados por la verdad para abandonarlo desengañados, o sea, para marcharse juntos con la verdad antes desechada pero ahora compartida.

Vicente Cabrera

Colorado State University.

\title{
NOTA BIBLIOGRAFICA SOBRE SOR JUANA INÉS DE LA CRUZ: SON TRES LAS EDICIONES DE BARCELONA, 1693
}

Pedro Henríquez Ureña, en su "Bibliografía de Sor Juana Inés de Ia Cruz", $R H i, 49$ (1917), 161-214, nos da en la p. 192, núm. 27, la descripción de un ejemplar del t. 2 de las obras de Sor Juana publicado en Barcelona el año de 1693. Henríquez Ureña sabía de la existencia de cinco ejemplares: tres de la Biblioteca Nacional de México (BNMx), uno de la Hispanic Society de Nueva York (HSNY) y otro de la Biblioteca Pública de Nueva York (PLNY). No sabemos a cuál de ellos se refiere Ia descripción que da en su artículo. Henríquez Ureña desconocía la existencia de otra edición de ese mismo lugar y año, cosa que descubrió Dorothy Schons al notar que su ejemplar y el de su amiga eran diferentes. En su Bibliografia de Sor Juana Inés de la Cruz, México, 1924, p. 34, nota, dice:

Debería hacerse mención de otra edición de 1693, del tomo II de sus obras completas. Henríquez Ureña únicamente cita una. Cuando menos hubo otra más. Al comparar una que tengo en mi poder con otra, propiedad de la señorita Goff, de Austin, Texas, se observan muchas dife-

4 En su reseña sobre El casamiento engañoso y Coloquio de los perros. Le marriage trompeur et Colloque des chiens, ed. por Maurice Molho; HR, 41 (1973), p. 563. 
rencias. Las carátulas son idénticas, excepto la clase y tamaño del tipo de letra y adorno tipográfico. Hay una pequeña diferencia en el tamaño de los volúmenes: el mío es de $151 / 2 \times 20 \mathrm{~cm}$., y el otro tiene $141 / 2 \times 20 \mathrm{~cm}$. (El descrito por Henríquez Ureña tiene $15 \times 20 \mathrm{~cm}$.). Ambos textos difieren en los adornos tipográficos, tamaño y clase del tipo de letras, ortografía, etc. Ignoro si la edición que describe Henríquez es idéntica a alguna de éstas.

Ermilo Abreu Gómez, Sor Juana Inés de la Cruz: bibliografia y biblioteca, México, 1934, registra dos ediciones, la citada por Henríquez Ureña (núm. 6, pp. 20-22) y la de D. Schons (núm. 7, pp. 22-24). No investigó los problemas de precedencia: simplemente llamó "la edición" a la comentada por Henríquez Ureña y " 2 a edición" a la que Schons consignó luego como diferente de aquélla. No sabemos si Abreu Gómez conocía el ejemplar que sirvió para la descripción de Henríquez Ureña. En las pp. 23-24 de su libro señala algunas de las diferencias entre la "edición Ureña" y la "edición Goff", pero no aclara el asunto debidamente. Como ejemplares de la "edición Ureña" enumera los de la PLNY y de la $H S N Y$, más cinco de México (p. 21). Al hablar de la "edición Goff" da, al final, el ejemplar Goff y el de Schons como pertenecientes a la misma edición, afirmación extraña, puesto que justamente Schons decía que eran distintos, y además ignoraba si el ejemplar de Henríquez Ureña era igual a alguno de los que ellas tenían ${ }^{1}$. Yo llamaré "ejemplar H. Ureña" al descrito por este crítico en el artículo citado; "ejemplar Goff" a aquél cuyas diferencias tipográficas apunta Abreu Gómez en su libro (pp. 23-24); y "ejemplar Schons" al que posee esta investigadora, y del cual habla en la nota citada pero sin hacer su descripción.

En el verano de 1969 examiné en la Biblioteca Nacional de Madrid (BNM) dos ejemplares (R-19234 y R-19447) impresos en Barcelona, 1693, y noté que eran diferentes. Se comprobaba así la aseveración de D. Schons de que "cuando menos hubo otra [edición] más". Dos años más tarde, cuando volví a la $B N M$ durante el verano de 1971 , al consultar el catálogo de la Sección Bibliográfica, hallé tres ejemplares más de esta edición: R-19237, R-17534 y R-17568². El primer ejemplar de

1 Méndez Plancarte en su Sor Juana Inés de la Cruz. Obras completas, t. 1, no dedica atención especial a la bibliografía de la poetisa. En la nota 59 a su "Introducción" (p. Ixii) se refiere someramente a las ediciones de Ios ts. 1,2 y 3 que se publicaron, especialmente a aquéllas que aparecieron en vida de la poetisa. En esa cita se refiere, al hablar del t. 2, a una sola edición de Barcelona, 1693, sin hacerse eco del descubrimiento de D. Schons. En la edición de Primero sueño de la Facultad de Filosofía y Letras de Buenos Aires, dos de sus principales colaboradores -G. Motdenhaver en su artículo "Observaciones críticas para una edición definitiva del Sueño de Sor Juana Inés de la Cruz", BdFS, 8 (1954-55), 293-296 y J. C. Merlo en su edición Sor Juana Inés de la Cruz, Barcelona, 1968, p. 71-, tienen en cuenta dos ediciones distintas de Barcelona, 1693: las identifican con B y b (o "ediciones 2: y 3:") , considerando más tardía a la que presenta más errores.

$2 \mathrm{Mi}$ gratitud al profesor E. M. Wilson por sus valiosas explicaciones y consejos, al señor F. Maldonado por haberme acompañado un día en Madrid (enero de 1972) a la Biblioteca Nacional para examinar los ejemplares de la edición de Barcelona, 1693, y por su ayuda posterior, y por último a mi marido E. L. Rivers, 
este grupo resultó ser igual al R-19234, y el segundo igual al R-19447. El tercer ejemplar, R-17568, no era idéntico a ninguno de los dos pares mencionados. Las diferencias comienzan con la portada y siguen a través de todo el libro. Para mayor claridad en la exposición, separé por grupos estos cinco ejemplares: a) R-19447, R-17534; b) R-19234, R-19237; c) R-17568. Me apresuro a decir que el material (composiciones, textos...) de estos tres grupos es el mismo. Las diferencias son pequeñas variantes textuales y de impresión: tipos de letra, dibujo y ortografía varían en los ejemplares pertenecientes a cada uno de los tres grupos. El grupo $a$, por otra parte, tiene tres páginas más que $b$ y $c$. Siguen los tres a la edición de Sevilla de 1692 -única del tomo segundo anterior a las de Barcelona- con las supresiones y adiciones ya señaladas por Henríquez Ureña en su artículo (pp. 192-193).

Damos a continuación la descripción de cada uno de estos grupos. En $a$ dice la portada:

SEGUNDO TOMO/DE LAS OBRAS/DE SOROR/JUANA INES/ DE LA CRUZ,/MONJA PROFESSA EN EL MONASTERIO/DEL SENOR SAN GERONIMO/DE LA CIVDAD DE MEXICO./ANTADIDO EN ESTA SEGVNDA IMPRESSION/POR SV AVTORA./Año [viñeta triangular de cinta entrelazada con hojas], 1693./[filete entrecortado (10) ] Impresso en Barcelona, por Joseph Llopis. $Y$ à su costa./Volumen en 4\%. 943 $, \mathrm{A}-\mathrm{Z}^{4}, \mathrm{Aa}-\mathrm{Zz}^{4}$, Aaa-Nnn4, Ooo2. Pp. (i-viii) 1-465 246 467470 pp. (i-vi) $=$ viii $470+$ vi $\mathrm{pp}$.

Contenido de los preliminares:

9r. Portada. 9v. Página en blanco. 92r-v. CENSVRA DEL R.mo P. MAESTRO/Iuan Navarro Velez, ...

Contenido de las páginas numeradas:

Crisis, sobre vn Sermon... 1-34; Poesias lirico-sacras 35-77; Poesias comico-sacras 78-170: Loa, que celebrando la concepcion de Maria Santissima... 78-85, Loa, ... El martyr del Sacramento 86-93; El Martyr del Sacramento/S. Hermenegildo/, Avto historial alegorico/Interlocutores./etc. 94-130; Loa,/para el avto intitulado,/El cetro de Joseph./131138; El cetro de Joseph 139-170; Poesias/liricas/[línea ornamental]/ 171-274: Primero sueño,/qve assi intituló ... 171-200; Poesías comicas /[línea ornamental]/275-456: Loa/a los años del Rey ... 275-284; Loa/

quien fue el que me puso en contacto, por primera vez, con los ejemplares registrados en la Sección Bibliográfica de la misma biblioteca (antes había visto sólo los "raros" catalogados en la Sección General). Agradezco, también, la ayuda y facilidades prestadas por las siguientes personas y entidades: el Director de la Biblioteca Capitular y Colombina de Sevilla; Sor Asunción del Convento de Sta. Paula, en Sevilla; la Directora de la Biblioteca de la Universidad de Granada; Biblioteca de la Universidad de Sevilla; Hispanic Society y Public Library of New York. Cualquier deficiencia en la exposición de este trabajo es, desde luego, responsabilidad mía.

3 En la descripción de los textos, al hablar de los folios el "9" ocupa el lugar de los "calderones". 
a los años de la Reyna Madre ... 285-291; Ecomiastico poema/a los años de la.../Condesa de Galve ... 292-301/Loa/a los años del... Conde de Galve./Que parece precediò á la Comedia ... 302-312; Amor es mas labirynto 313-373; Loa/qve precedio a la comedia, que se sigue 374-384; Los empeños de vna casa 385-449; Sarao/de Qvatro Naciones 450-456; Mas poesias/lirico-sacras 457-470.

$\mathrm{Nnn}_{4}{ }_{-} \mathrm{Ooo}_{2}{ }^{\mathrm{r}}$. Indice (seis folios sin numerar. El verso de $\mathrm{Ooo}_{2}$, en blanco). Los tres grupos $a, b$ y $c$ siguen la misma foliación y paginación y tienen los mismos textos página por página hasta la p. 449. A partir de la p. 450, en la cual, en los tres grupos, comienza el "Sarao de Qvatro Naciones", en $b$ y $c$ se abrevia el número de páginas porque la tipografía es más pequeña y hay menos espacio entre los títulos y al comienzo de las composiciones. Resultan entonces tres páginas menos que en $a$ (470 pp. en $a$ y $467 \mathrm{pp}$. en $b$ y $c$ ). La descripción para $b$ y $c$ será igual que para $a$ excepto: Sarao/de Quatro Naciones 450-455; Mas poesias/lyrico-sacras $456-467$.

El índice es el mismo con igual número de páginas. Los ejemplares de $a$ son de $12.2 \times 16.9 \mathrm{~cm}$. El ejemplar R-17534 está encuadernado en piel roja con grabados en dorado, canto dorado y grabado. En la portada tiene un sello con las iniciales BR entrelazadas. El ejemplar R-19447 está encuadernado en pergamino; el canto debió ser rojo, hoy bastante desleído. Sobre la fecha, en la portada, hay un sello redondo con grabado de una cruz y leyendas en latín, lo cual dificulta ver la fechat. Grupo $b$. Portada: SEGUNDO TOMO/DE LAS OBRAS/DE SOROR/ JUANA INES/DE LA CRVZ,/MONIA PROFESSA EN EL MONASTERIO/DEL SENTOR SAN GERONIMO/De la Ciudad de Mexico./ ANADIDO EN ESTA SEGVNDA IMPRESSION/POR SV AVTORA. /Año [viñeta: frutero lleno de frutas y hojas. Dos pájaros a cada lado comiendo de las frutas]. 1693./CON LAS LICENCIAS NECESSARIAS. /[filete entrecortado (10?)]/Impresso en BARCELONA: POR JOSEPH LLOPIS./Y à su costa./

Volumen en 40.94, A $-\mathrm{Z}^{8}, \mathrm{Aa}-\mathrm{F}_{\mathrm{f}}^{8}, \mathrm{Gg} 4$. Pp. [i-viii] 1-360 465362 467 pp. $[\mathrm{i}-\mathrm{vi}]=$ viii $+467+$ vi pp. $12.2 \times 17.7 \mathrm{~cm}$. Los ejemplares de este grupo, R-19237 y R-19234, están encuadernados en pergamino, cantos blancos, y el papel parece de inferior calidad que el utilizado en $a$ y $c$. El ejemplar R-19234 tiene, en el índice, correcciones a mano que remiten a las páginas donde realmente se hallan las poesías indicadas. El Índice se imprimió con errores y el dueño de este ejemplar hizo las correcciones necesarias.

Grupo $c$. Portada: SEGUNDO TOMO/DE LAS OBRAS/DE SOROR /JUANA INES/DE LA CRUZ,/MONJA PROFESSA EN EL MONASTERIO/DEL SENOR SAN GERONIMO/De la Ciudad de Mexico./ AÑADIDO EN ESTA SEGUNDA IMPRESSION/POR SU AUTORA.

\footnotetext{
4 He preferido, por ese motivo, utilizar xerocopia de uno de los ejemplares que hay en la Biblioteca Capitular y Colombina de Sevilla donde se ve claramente la fecha y demás detalles de la portada.-El profesor E. M. Wilson ha tenido ba bondad de enviarme la descripción del ejemplar de Barcelona 1693 que posee cotejado con el de $U L C$ (ambos, a).
} 
/Año [viñeta: frutero lleno de frutas, flores y hojas]. 1693./CON LAS LICENCIAS NECESSARIAS./[filete entrecortado (10?)]/Impresso en BARCELONA: Por JOSEPH LLOPIS./Y á su costa./

Volumen en 49 94, A - Z8, Aa - F8, Gg4. Pp. [i-viii] 1-36 57 38-122

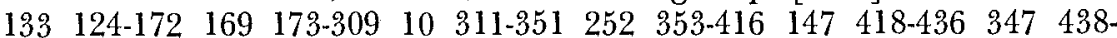
467 pp. $[\mathrm{i}-\mathrm{vi}]=$ viii $+467+$ vi pp. $12.1 \times 17.5 \mathrm{~cm}$. Este ejemplar tiene exactamente el mismo contenido que $b$. Las mismas páginas remiten exactamente a los mismos textos línea por línea. Está encuadernado en piel, canto rojo y lomo con letras y adornos en dorado: EL FEN./DE/ $\mathrm{MEXICO/T}$. II. Es el segundo volumen de una colección de los tres tomos de sor Juana que perteneció a Pascual Gayangos, cuyo sello en rojo aparece en la portada.

\section{VARIANTES DE IMPRESIÓN}

P. 1: El filete y adorno de la parte alta de la página es diferente en cada edición; la $A$ del reclamo ocupa en cada edición un lugar diferente bajo la palabra "alabando"; la $M$ al comienzo de la "Crisis..." es diferente en tamaño y adorno en las tres ediciones $\|$ p. 34: Adorno a pie de página: sol rodeado de estrellitas y orlas con inscripción en latín $a$; adornos geométricos formando cuadritos $b$; pájaro sobre un zócalo $c \|$ p. 36 : cesta con flores $a$; adorno muy parecido al de la p. $34, b$; tres angelitos $c \|$ p. 40: adorno triangular $a$; idéntico al que cada uno tiene en la p. $36 b$ y $c \| \mid$ p. 50: nada, $a, b$; adorno $c||$ p. 57: Signatura H. $a$; nada, $b, c \|$ p. 60: LETRA XXVJ $a$; letra XXVJ, $b$; letra XXVI, $c$ \| 65: Villancicos, / con que [que, $c$ ] se solemnizaron en la/Santa Iglesia, y primera Cathedral de la Ciudad de Ante-/quera [Antequera, b], Valle de Oaxaca, los Maytines de la Gloriosa Martyr $/ \ldots[$ Mar- $/ \ldots, c] \ldots$ || p. 75: nada $a$; tres grupos de tres estrellitas $b$; un grupo de tres estrellitas entre paréntesis $c \|$ p. 77: nada $a$; adornos distintos en $b$ y $c$ $\|$ p. 85: nada, $a$; cinco [tres, $c$ ] adornos geométricos cuadrados, $b$; signatura LOA, $a ; \mathrm{F}_{3} \mathrm{LOA}, b, c \| \mid$ p. 86: Comienza la Loa a San Hermenegildo. La línea ornamental, que separa título e "Interlocutores" del texto, diferente en $a, b, c \|$ p. 87: Signatura De, $a$; $\mathrm{F}_{4} \mathrm{De}, b, c \|$ p. 94: tipo de letra diferente en $a, b, c$; el encabezamiento difiere en $a, b, c$. Véase $R$ de Historial y Alegórico, y en el texto, también diferente, véase la $H$ inicial del primer verso || p. 130: frutero con flores, frutas y dos pájaros, $a$; quince adornos geométricos cuadrados que forman un triángulo, $b$; gran adorno: cabeza coronada por una cesta de frutas, dos figuras a los lados cogiendo de ellas, dos cabezas a los lados con hojas en la boca, dos cabezas de animales, $c \|$ p. 138: adorno en forma triangular (igual al de p. 40) $a$; adorno formando pequeños cuadrados $b$; tres cabezas de angelitos $c \|$ p. 170: adorno triangular (como p. 40) a; diez adornos geométricos cuadrados $b$; flores, plumas, repisa con tres angelitos $c$ \| p. 171: Comienzan las "Poesías liricas". Tipo y tamaño de letra diferente en $a, b, c \|$ p. 200: al final del Sueño, reproduce el adorno de su respectiva p. 130, $a, b, c||$ p. 216: nada, $a$; cuatro pequeños cuadrados $b, c$ [el del extremo derecho al revés $c$ ] || p. 226: cruz 
central con ornamentos laterales diferentes en $a, b, c \|$ p. 232: letra más pequeña, $a ; \mathrm{Vn}[\mathrm{Un}, c]$ festejo... y [Virreyna/a] Virreyna de Mexico/ $\ldots a, b, c \|$ p. 235: Signatura: $\mathrm{Gg}_{2} \mathrm{Y}$ assi, $a$; Y $b, c \|$ p. 239: adornos diferentes en $a, b, c \|$ p. 240: nada, $a$; seis [cuatro, $c$ ] adornos geométricos cuadrados, $b, c \|$ p. $250 a(251 b, c):$ los adornos son distintos en $a, b, c \|$ p. 275: Ibid., $a, b, c$; Nv[u,c]estro, $a, b, c \|$ p. 284: adornos diferentes en $a, b, c \|$ p. 291: adorno en forma triangular como el de p. 40, $a$; veintiún dibujos en cuadritos $b$; adorno de tallos y flores que rematan en flor de lis, invertido, $c||$ p. 312: adorno triangular igual al de la portada, $a$; veintiún dibujos en cuadrados que forman un gran triángulo $b$; dos angelitos sobre una repisa, dos pájaros y ornato de plumas, $c \|$ p. 384: los adornos difieren en $a, b, c \|$ p. 385: Interlocutores, $a$; INTERLOCU[V, $c$ ] TORES, $b, c \|$ p. $450 a(449 b, c)$ : tipo de letra más grande, $a$; $\mathrm{QV}[\mathrm{U}, c]$ ATRO $a, b, c \|$ p. $456 a(455 b, c)$ : seis figuras geométricas formando un triángulo, $a$; nueve adornos geométricos en dos líneas, cinco en la superior, cuatro en la inferior, $b$; cara de angelito rodeado de un ornamento de plumas $c \|$ p. $457 a(456 b, c)$ : Comienzan "Mas poesías li $[\mathrm{y}, b, c]$ rico-sacras" $a, b, c$; tipo de letra distinto en $a$; la misma tipografía $b, c \|$ p. $470 a(467 b, c)$ : mismo adorno de p. 456, $a$; nada $b, c$.

Terminan aquí las muestras de las variantes en la impresión. Para las diferencias textuales tendré en cuenta, además de $a, b$ y $c$ la edición de Sevilla, 1692, $(A)$ pues luego trataré de establecer la precedencia entre los tres grupos basándome solamente en las diferencias textuales; como ya he dicho, la edición de Sevilla, 1692, es la única anterior a la de Barcelona, 1693.

En la $B N M$ hay dos ejemplares de la edición de Sevilla: R-19244 y R-19252. El primero está completo; al segundo le faltan las diez primeras páginas, cuatro numeradas al final (pp. 543-546), más el índice (6 pp.). Para la descripción que sigue utilicé el ejemplar de la PLNY. La portada de esta edición está impresa en negro y rojo y es como sigue: SEGVNDO VOLVMEN/DE LAS OBRAS/DE SOROR/JVANA INES/DE LA CRVZ,/MONJA PROFESSA EN EL MONASTERIO/ DEL SENTOR SAN GERONIMO/DE LA CIVDAD DE MEXICO, /DEDICADO POR SV MISMA AVTORA/A D. JVAN DE ORUE/Y ARBIETO/CAVALLERO DE LA ORDEN DE SANTIAGO./Año [viñeta de hojas entrelazadas en forma triangular] 1692. [Filete entrecortado (11)]/Con Privilegio, En Sevilla, por TOMAS LOPEZ DE HARO,/Impressor, y Mercader de Libros.

Volumen en 40, 94, b $-\mathrm{m}^{4}, \mathrm{n}^{2}, \mathrm{~A}-\mathrm{Z}^{4}, \mathrm{Aa}-\mathrm{Zz}^{4}, \mathrm{Aaa}-\mathrm{ZzZ}^{4}$. Pp. (i-c) 1-69 [en blanco] 71-546 pp. [i-vi] $=c+546+$ vi pp. Para la descripción del contenido, véase Henríquez Ureña, art. cit., pp. 186-191.

Este ejemplar de la $P L N Y$ tiene dos grabados, uno de los cuales no he visto en los otros ejemplares de esta edición. El primero, hoja inserta entre la portada y el comienzo del texto (dedicatoria a Orúe y Arbieto), es un escudo enmarcado en ornato de plumas, una armadura coronada de plumas, flores de lis y cintas delgadas por debajo. El escudo está dividido en dos partes. La superior presenta tres barras con un árbol 
donde apoya sus patas delanteras un animal que parece un ciervo. En la parte inferior hay dos árboles más pequeños y en medio cinco dibujos que parecen corazones. El otro grabado -inserto inmediatamente antes de empezar la Crisis (p. 1)- de D. Lucas de Valdés, es un retrato de sor Juana a cuyos lados están Mercurio y Marte sosteniendo una corona de laurel sobre el retrato de la poetisa, y en la parte superior la Fama con dos trompetas; sus alas están cubiertas de pequeños ojos y oídos ${ }^{\mathrm{s}}$.

\section{VARIANTES TEXTUALES}

f. $4 \mathrm{r}, A$ (f. $3 \mathrm{r} a, b, c$ ) : escriptos $A, a$; escritos, $b, c \mid$ de esta $A$, $a$; desta, $b, c \|$ p. 2: va-/sas, $A, a$; basas, $b, c \mid$ ya, $A, a, b$; yá, $c \mid$ dexarà, $A, a, b$; dexaré, $c \mid$ accepcion, $A, a, b$; acepcion, $c \mid$ Sujeto, $A$, a; Sugeto, $b, c \|$ p. 3: de este, $A, a$; deste, $b, c \|$ p. 6: finezas, $[; c] A, a, b, c \|$ p. 9: aumentò $A, a$; ausenta, $b, c \|$ p. $85 A$ (p. $45 a, b, c):$ Impi[y, $a]$ reo, $A, a$; Empyreo, $b$; Epyreo, $c$; $\|$ p. $121 A(94 a, b, c):$ consonancias $[? b, c]$ $A, a, b, c \|$ p. $157 A(130 a, b, c): \mathrm{t}[\mathrm{T}, b, c] \mathrm{ierra}, A, a, b, c \| \mathrm{p} .158 A$ $(131 a, b, c)$ : assi $[, b, c], A, a, b, c \|$ p. $292 A(216 a, b, c)$; 'q he [que he $b$ ] trazado, $A, a, b, c \mid$ 'q os cause [que cause, $b, c]$ espanto [; $A, a, b]$ $A, a, b, c \mid$ 'q [que, $b$ ] indice infiero, $A, a, b, c \mid \mathrm{C}[\mathrm{c}, b, c]$ imero, $A, a$, $b, c \|$ p. $307 A(231 a, b, c): \operatorname{Excma~(mo)~} A$, $a$; excelentissima (ssimo), $b, c \mid$ Hendecasyllabo, $A$, $a$, endecasilabo, $b, c \|$ p. $388 A(312 a, b, c)$ : Occidental[Occidétal, $a]$ Orbe, $A, a, b, c \mid[\mathrm{P}, A]$, populares voces, $A, a$, $b, c \mid$ Desseaba, A: Deseaba, $a, b, c \|$ (signatura y palabra al comienzo de la pág. sig.) . p. $429 A$ (353 $a, b, c)$ : Page [Paje, $b]$, / Page, $A, a, b$; no hay, $c \| \mid$ p. $532 A(456 a, b, c):$ Ya, 'q [que, $b, c]$ las demo[ó, $b, c]$ straciones, $A, a, b, c \mid$ Táto, $A, a, c$; Tanto $b$; $\mid \mathrm{A}[\mathrm{a}, a]$ mor, $A, a, b, c$ $\mid$ in[m, $A$ ]menso, $A, a, b, c||$ p. $544 A(468 a, b, c)$ : DEZIMAS [QVINTILLAS, $b, c] \mid$ Geronimo Meditaba, $A, a, b, c||$ p. $546 A(470 a, 467$ $b, c)$ : SONETO A $[\mathrm{AL}, b, c]$ Señor S. [SAN, $b, c]$ Joseph [JOSEPH, $b, c] A, a, b, c \mid$ Aveja $A, a$; Avexa, $b, c[[\mathrm{P}, A] \mathrm{palma}, A, a, b, c \mid$ Assi Rosa [, $A, a]$ María [, $A, a] A, a, b, c \mid$ Tirano, $A$; tyrano, $a, b, c \mid$ Solo vos (ó Joseph !), $A$; (ó Joseph [!, $a] a, b, c \|$ El índice de $A$ es distinto de $a, b, c$ que lo tienen igual; p. iv: Hirió, $a, b$; Hi, $c$; nada en $A$. tuales:

Resultados de estos ejemplos (tomados al azar) de variantes tex-

1) Número de veces en que estos grupos presentan casos aislados: A $8, a 4, b 5, c 7$.

2) Número de veces en que presentan incidencia por pares y tríos: $A a$ 19, $a b$ 1, $b c$ 19; $A a b$ 5, $A a c$ 3, $A b c$ 2, $a b c 5$.

3) Número de veces en que los pares que siguen aparecen en cualquier combinación que presenta la misma grafía: $A a 26, a b 5, A c 5, a b 10$, ac $7, b c 24$.

En los tres resultados hay una progresión en los cambios que va

5 En la edición separada de El sueño de Méndez Planearte, inmediatamente antes de las "Notas textuales" hay una lámina que reproduce este grabado. 
de $A \rightarrow a \rightarrow b \rightarrow c$. Si nos fijamos en la lista de resultados 1 , vemos que $A$ tiene el mayor número de casos aislados ya que es edición de otro lugar y año que sirvió de base para las siguientes. Siguen $a$ con 4 y $b$ con 5 . El grupo $c$ es el que, de las ediciones de Barcelona 1693, presenta más casos aislados. Creo que estas diferencias textuales, más las de impresión que vimos antes, son suficientes para afirmar la existencia de tres ediciones diferentes, constituidas por los grupos $a, b$ y $c$.

En la lista de resultados $2 A$ y a presentan 19 casos con la misma particularidad textual; lo mismo ocurre con los grupos $b$ y $c$. Los tríos $A a b$ y $a b c$ presentan el mismo número de incidencias, 5 eñ cada caso. Las otras combinaciones señalan escasas incidencias (l a 3 ). Obviamente el $a$ sigue muy de cerca a $A$ y lo mismo ocurre a $c$ con respecto a $b$. En la lista de resultados 3 , el número de veces en que $A$ y $a$ presentan la misma grafía es el más alto, 26. Los grupos $b$ y $c$ presentan 24 casos de incidencia; $a$ y $b, 10$. Estos tres últimos casos me reafirman en la creencia de que $a$ es la la edición de Barcelona 1693 por ser la más cercana, textualmente, a la de Sevilla del año anterior. Además, $a$ es edición más cuidadosa que $b$ y $c$. Véanse los versos completos de algunos ejemplos que di para las diferencias textuales: "Y no es bien, 'q os cause espanto;" (a) contra "Y no es bien, que cause espanto;" (b y $c$ ) p. 216; "Assi Rosa, Maria, mas hermosa" y "Solo vos (ò Joseph!) vais a esconderlo;" de a (p. 470) contra "Assi Rosa Maria mas hermosa" y "Solo vos (o Joseph) vais a esconderlo;" de $b$ y $c$ (p. 467). Véanse también los ejemplos de pp. 353 s.: Page,/Page, de $a$ contra Paje,/Page, de $b$ y nada en $c$; el de la p. 9 "ausentò", $a$, que es la lectura correcta contra "ausenta" de $b$ y $c$. Además, el grupo $a$ es la edición que más se parece en los arreglos tipográficos a la de Sevilla. (Véase el adorno de la portada y la última página numerada de cada grupo, como ejemplos) .

La $2^{\text {a }}$ edición de Barcelona sería $b$. Por el número de incidencias $(10)$ entre $a$ y $b$, vemos que el grupo $b$ es el que sigue a $a$. El ejemplar $c$, que presenta 24 incidencias con $b$, constituirá la 3 a edición del mismo lugar y año. Las tres cifras, $26(A a), 10(a b)$ y $24(b c)$ nos dan la clave. Las otras combinaciones $(A b, 5 ; A c, 5$ y $a c 7)$ nos marcan la distancia que dentro de una base común van presentando los grupos. El más alejado es el de Sevilla; Ias tres ediciones de Barcelona son muy parecidas pero no idénticas. Presentan los mismos textos, y la $2^{\mathrm{a}}$ y $3^{\mathrm{a}}$ tienen exactamente la misma tipografía e igual número de páginas; la la, con tres páginas más, se acerca más a la edición que se tomó como modelo. La fama de sor Juana en España hizo posible este fenómeno: el t. 2 de sus obras fue publicado primero en Sevilla en 1692, tres veces en Barcelona en 1693, en Madrid en 1715 y 1725. Su t. 1 fue publicado nada menos que nueve veces y el t. 3, cinco.

Pasamos ahora a la identificación del "ejemplar Ureña", del "ejemplar Goff" y del "ejemplar Schons" de que hablamos antes.

Las tres ediciones tienen casi la misma portada excepto por la tipografía y el adorno. Usan las mismas palabras en el título desde "Segundo" hasta "Autora". Hay diferencia en la $u$ de "Cruz", "Ciudad", "Segunda", "Su" y "Autora": $a$ tiene $u$ en "Cruz" y $v$ en las otras pala- 
bras; $b$ tiene $u$ en "Ciudad" y $v$ en el resto de las mencionadas, y $c$ tiene $u$ en todas. Henríquez Ureña (op. cit., pp. 192-195) no describe el adorno (ignoraba que hubiera más de una edición) pero, al describir la portada, sí anotó la $u$ en todas esas palabras (en otras ediciones hace la diferencia); por lo tanto podemos concluir que esa descripción pertenece a un ejemplar del grupo $c$, $3^{a}$ edición.

Abreu Gómez (op. cit., pp. 23-24) compara la "edición Ureña" con la "edición Goff". Al hablarnos de sus diferencias describe algunos de los adornos de esta última. Si, efectivamente, el autor se refiere al ejemplar de Goff, podemos, a través de estas explicaciones, identificarlo con una de nuestras ediciones. Pongo entre paréntesis la descripción que me pertenece. En efecto, Abreu Gómez dice que en la p. 34 hay "un pájaro" ("pájaro sobre un zócalo"); el de la p. 36 lo describe como "tres ángeles" ("tres angelitos"); dice del de la p. 40 que es "igual" [al anterior], (repetí "tres angelitos"); describe el de la p. 85 como "adorno convencional", (expliqué "tres adornos geométricos, cuadrados"); dice del de la p. 190 "adorno grande (figuras de ángeles)", ("gran adorno: cabeza sosteniendo cesta de frutas", etc.); del adorno de la página 138 nos dice: "tres ángeles" ("tres cabezas de angelitos") ; del de la p. 170 "adorno especial: querubines, flores, etc." ("adorno de flores y plumas, repisa con tres angelitos"); del de la p. 200 dice "igual al de la 130" ("el adorno de la p. 130"); del de la p. 226 "convencional" ("adorno de cruz central con dibujos a los lados, ..."). El adorno de la p. 291 lo explica así: "adorno convencional grande" ("gran adorno..."); el de la p. 312 "adorno grande con querubines y pájaros" ("dos angelitos sobre una repisa, dos pájaros..."). Creemos que estas coincidencias son suficientes para clasificar el ejemplar de la "edición Goff", comentado por Abreu Gómez, con el grupo 3a edición de Barcelona 1693, ya que las explicaciones nuestras pertenecen a ese grupo $c$. Es decir que el "ejemplar H. Ureña" y el "ejemplar Goff" serían idénticos.

En cuanto al "ejemplar Schons" sabemos por ella misma (op. cit., p. 34) que su ejemplar tiene la portada igual al de la señorita Goff excepto por el adorno y tipo de letra. Son los grupos $b$ y $c$ los que presentan esta semejanza estrecha. (El grupo $a$, además de adorno y tipos de letra diferentes, no tiene, después del año, "Con las licencias necessarias." de $b$ y $c$, y tiene al final en una misma línea "Impresso en Barcelona, por Joseph Llopis. $Y$ a su costa", mientras que $b$ y $c$ separan en otra línea "Y a su costa", y no está en cursivas). Podemos concluir que el "ejemplar Schons" pertenece al grupo $b, 2^{3}$ edición de Barcelona, 1693.

Abreu Gómez tiene al final de su libro (p. 425) "fotograbados de las portadas de las principales ediciones de Sor Juana". El noveno de esos fotograbados nos presenta una portada perteneciente a $c$, nuestra 3 a edición de Barcelona, 1693. Los ejemplares de la HSNY y de la PLNY pertenecen también, los dos, a la que llamamos tercera edición.

La edición mencionada del Sueño (cf. supra, nota 1) de la Facultad de Filosofía y Letras de Buenos Aires (p. 84) tiene la descripción de 
los dos ejemplares de Barcelona, 1693, que se utilizaron en ella. El marcado con $B$ corresponde a un ejemplar de nuestro grupo $b, 2^{a}$ edición; el marcado con $b$, a uno de nuestro grupo $c$, 3a edición ${ }^{6}$.

Sabemos que existen los siguientes ejemplares de las ediciones del t. 2, de 1693 que aquí tratamos ${ }^{7}$ :

1*a edición: $B N M, 2 ; B C C S, 2 ; B U G, 1 ; B C S P S, 1 ; B N P, 1 ; U L C, 1$; $E M W, 1 ; B P L, 1 ;$ total, 10.

2a edición: $B N M, 2$; Goff (?), 1; $B C B A, 1^{8}$; total, 4.

3 edición: $B N M, 1 ; B C C S, 1 ; H S N Y, 1 ; P L N Y, 1$; Schons (?), 1; Ejemplar del fotograbado de Abreu Gómez, 1; Microfilm en $L C W, 1^{9}$; Descripción de $b$ en la edición del Sueño de la F.F.L.B.A., ${ }^{10}$; total, 8.

Esta tercera edición tuvo, al parecer, más difusión en América mientras que la primera fue quizá más conocida en Europa. Henríquez Ureña (op. cit., p. 192), repetimos, habla de cinco ejemplares, tres de ellos de la $B N M x$, y los otros dos de la $H S N Y$ y de la $P L N Y$. No conozco los ejemplares de México, pero si Henríquez Ureña los vio pertenecen,

6 G. Moldenhauer, art. cit., pp. 295-296 se refiere al hecho de que Méndez Plancarte no distinguiera entre las dos ediciones conocidas en Barcelona, 1693, lo que traería "la mayoría de sus desaciertos... así como... cierta fluctuación de sus decisiones". Hay, en efecto, lectura distinta en los versos del Sueño que Moldenhauer cita, lectura que se encuentra en las ediciones que llamamos $2^{\mathrm{a}}$ y $3^{\mathrm{a}}$ ( $b$ y $c$ ), $B$ y $b$, respectivamente, de la edición argentina. Méndez Plancarte utilizó un solo ejemplar de Barcelona, 1693, y lo llamó $B$, lo cual no quiere decir que fuera igual al $B$ de la edición de Buenos Aires. Por las explicaciones del crítico mexicano de esos versos del Sueño (op. cit., pp. 578-581), sabemos que su ejemplar pertenecía al grupo $c$ (3: edición nuestra). Pero Méndez Planearte cotejó, también, un ejemplar de la edición Sevilla, $1692(A)$, y en casi todos esos versos, $a$ y $b$ presentan la misma lectura que $A$; por lo tanto, pudo hacer casi siempre las correciones necesarias aunque no dispusiera de ejemplares pertenecientes a ninguna de esas dos ediciones anteriores.

7 Utilizo las siguientes siglas: $B N M=$ Biblioteca Nacional de Madrid; $B C C S=$ Biblioteca Capitular y Colombina de Sevilla; $B C B A=$ Biblioteca del Congreso, Buenos Aires; $B U G=$ Biblioteca de la Universidad de Granada; $B C S P S=$ Biblioteca del Convento de Santa Paula, en Sevilla; HSNY $=$ Hispanic Society of New York; $P L N Y=$ Public Library of New York; $L C W=$ Library of Congress, Washington, D. C.; $\overline{U L C}=$ University Library of Cambridge; $E M W=$ E. M. Wilson; $B P L=$ Boston Public Library (Ticknor Collection). Desconozco la procedencia de este ejemplar, pero probablemente fue adquirido en Europa.

8 La edición del Sueño de la Facultad de Filosofía y Letras de Buenos Aires (p. 36) dice que "la primera edición fechada en Barcelona en 1693..." es decir la $B$, se conserva en la Biblioteca del Congreso de Buenos Aires. No dicen dónde se guarda la edición que llaman $b$.

9 En la Biblioteca del Congreso en Washington, se encuentra un "microfilm" (hecho en 1942) del original que pertenece a la Colección Medina de la Biblioteca Nacional de Chile; corresponde al grupo $c$, nuestra 3 a edición.

10 No consignamos el "ejemplar H. Ureña" porque, probablemente, su descripción es de un ejemplar de los de Nueva York. No consignamos tampoco el ejemplar cuyo fotograbado - perteneciente a esta 3: edición- aparece en el t. 3 de las Obras completas de Sor Juana, de Méndez Planearte (frente a la p. 502) porque no sabemos si es el mismo del fotograbado del libro de Abreu Gómez. En Genio y figura de Sor Juana Inés de la Cruz de RAMón XIRAU, aparece en las pp. 104-105 un fotograbado de un ejemplar también perteneciente a nuestra tercera edición. Xirau la llama "reedición de Barcelona, 1693". El ejemplar pertenece a la Colección de José Gargollo. Quizá sea el $b$ descrito en la edición del Sueño de F.F.L.B.A. 
seguramente, como los de Nueva York, a la 3a edición; como sabemos, el crítico dominicano conocía sólo una edición y por lo tanto todos los ejemplares que vio eran iguales. Abreu Gómez no se refiere en su libro más que a dos ediciones. En la $1^{\text {a }}$ edición (marcada con el núm. 6, pp. 20-21) consigna los 5 ejemplares que hay en México más los de la HSNY y PLNY mencionados antes por Henríquez Ureña. En la que llama segunda edición (marcada con el núm. 7) consigna los ejemplares de Schons y Goff, pero ya sabemos que estos dos ejemplares son distintos. Es de suponer que al escribir su libro tuviera los ejemplares de México a su alcance y consignara las diferencias, pero al referirse al núm. 6 y dar la lista de los ejemplares de México, no dice que haya diferencias entre ellos. Como he dicho, el fotograbado de la portada que aparece en su libro pertenece a un ejemplar de la 3a edición. Podríamos aventurar que la difusión geográfica de estas ediciones corroboraría nuestra tesis clasificadora en cuanto a la precedencia. $\mathrm{La} \mathrm{I}^{\text {a }}$ se agotaría en España, pasando luego a otros países europeos; de la 2a llegarían algunos a América y muchos más de la $3^{a}$, casi agotado ya el mercado español.

En todo caso, creo haber demostrado que en la ciudad mediterránea de Barcelona, lejos del golfo de México, en el año de 1693, se publicaron tres ediciones del Segundo Tomo de sor Juana Inés de la Cruz, homenaje al saber, lirismo y hermosura de la Musa Décima.

Western Maryland College.

Georgina SAbat de Rivers

\section{ACOTACIONES A EL JUGUETE RABIOSO DE ROBERTO ARLT}

El año de 1926 es de notable importancia para la narrativa argentina. En él aparecen Don Segundo Sombra, de Ricardo Güiraldes; Los desterrados, de Horacio Quiroga; y El juguete rabioso, de Roberto Arlt. La primera ofrece una original visión del mundo rural en la que coinciden lo tradicional y lo moderno. Sus páginas tienen, muchas veces exquisitez poemática. Se le han señalado muchos antecedentes, algunos innegables, pero lo cierto es que con Don Segundo Sombra se abre y se cierra un tipo de novela: el mundo que representa y la perfección que logra la hacen inimitable.

Horacio Quiroga (1878-1937), consagrado como cuentista excelente desde la publicación de sus Cuentos de amor, de locura y de muerte (1917) continúa su serie de pequeñas obras maestras que culmina con Los desterrados. Aquí vuelve sobre su tema reiterativo y siempre fecundo, que es la selva virgen e indómita, y la aventura, la soledad, la reflexión amarga, la esperanza angustiante que en ella aguardan al hombre. La influencia de Lugones, de Poe, de Maupassant, de Jack London, de Kipling, se resuelven en una original narración joven, producto de un artista maduro. Luego viene el silencio. Ni Más allá -la colección de 